\title{
Low Baseline Ischemic Water Uptake is Directly Related to Overestimation of CT Perfusion-Derived Ischemic Core Volume
}

Rosalie V. McDonough ( $\nabla$ r.mcdonough@uke.de)

University Medical Center Hamburg-Eppendorf

Sarah Elsayed

University Medical Center Hamburg-Eppendorf

Lukas Meyer

University Medical Center Hamburg-Eppendorf

Theresa Ewers

University Medical Center Hamburg-Eppendorf

Matthias Bechstein

University Medical Center Hamburg-Eppendorf

Helge Kniep

University Medical Center Hamburg-Eppendorf

Marie Teresa Nawka

University Medical Center Hamburg-Eppendorf

Tobias D. Faizy

University Medical Center Hamburg-Eppendorf

Gerhard Schön

University Medical Center Hamburg-Eppendorf

Götz Thomalla

University Medical Center Hamburg-Eppendorf

Jens Fiehler

University Medical Center Hamburg-Eppendorf

Uta Hanning

University Medical Center Hamburg-Eppendorf

Andre Kemmling

Westpfalz Klinikum

Gabriel Broocks

University Medical Center Hamburg-Eppendorf 
Keywords: Computed-tomography perfusion, acute ischemic stroke, EVT, core-overestimation, NWU Posted Date: November 1st, 2021

DOI: https://doi.org/10.21203/rs.3.rs-1013917/v1

License: (c) (i) This work is licensed under a Creative Commons Attribution 4.0 International License. Read Full License 


\section{Abstract \\ Background}

Computed-tomography perfusion (CTP) is frequently used to screen acute ischemic stroke (AIS) patients for endovascular treatment (EVT), despite known problems with ischemic "core" overestimation. This potentially leads to the unfair exclusion of patients from EVT. We propose that net water uptake (NWU) can be used in addition to CTP to more accurately assess the extent and/or stage of tissue infarction.

\section{Methods}

Patients treated for AIS between 06/2015-07/2020 were retrospectively analyzed. Baseline CTP-derived core volume (pCore) and NWU were determined. Logistic regression tested the relationship between baseline clinical and imaging variables and core-overestimation (primary outcome). The secondary outcomes comprised 90-day functional independence (modified Rankin score) and lesion growth.

\section{Results}

284 patients were included. Median NWU was 7.2\% (IQR:2.6-12.8). ASPECTS (RR:1.28,95\%Cl:1.09-1.51), NWU (RR:0.94,95\%Cl:0.89-0.98), onset to recanalization (RR:1.00,95\% $\mathrm{Cl}: 0.99-1.00)$ and imaging (RR:1.00,95\% Cl1.00-1.00) times, and pCore (RR:1.02,95\%Cl:1.01-1.02) were significantly associated with core overestimation. Core-overestimation was more likely to occur in patients with large pCores and low NWU at baseline. NWU was significantly correlated with lesion growth.

\section{Conclusion}

NWU can be used as a supplemental tool to CTP during admission imaging to more accurately assess the extent of ischemia, particularly relevant for patients with large CTP-defined cores who would otherwise be excluded from treatment.

\section{Introduction}

Advanced imaging is often used for the treatment selection of patients with acute ischemic stroke (AIS) due to large vessel occlusion (LVO) by identifying the volume of ischemic yet potentially salvageable tissue (penumbra). Despite recent breakthroughs in AIS therapy regimes with the extension of the acceptable time window for endovascular treatment (EVT), the dynamics of the infarct growth curve remain unknown ${ }^{1-3}$. It has been proposed that some patients are "fast progressors", presenting with large volume infarctions within a relatively narrow time window, while others retain tissue viability despite long 
vessel occlusion times (i.e., "slow progressors") ${ }^{4}$. In almost all cases, however, the vast majority will undergo irreversible tissue damage if left untreated ${ }^{4,5}$.

Certain computed tomography perfusion (CTP)-based parameters, such as cerebral blood volume (CBV) or cerebral blood flow (CBF) are frequently employed to assess the extent of irreversibly damaged tissue during admission imaging, particularly for those presenting within the extended time window ( $>6$ hours $)^{6}$. It is thought that the larger the ischemic lesion, as well as the smaller the penumbral mismatch, the riskier it is to perform an invasive procedure. Indeed, in the DAWN and DEFUSE III landmark trials, patients were stratified for treatment selection based on the CT or MR-derived size of the infarct core, the ratio of core to penumbra, and/or the mismatch between clinical severity and imaging results ${ }^{2,3}$. As a result, patients with large cores (e.g., potential "fast progressors"), as well as those with minimal clinical deficits (potential "slow progressors") were excluded from these studies. In fact, relatively little is known about the benefit of treatment in such patient groups.

Despite the consequences for individual patients, the question of validity of such methods remains ${ }^{7}$. In addition to providing limited spatial resolution, CBV and CBF have been repeatedly observed to overestimate final infarct size ("ghost infarct core" phenomenon), particularly within the early time window $^{8-10}$. Net water uptake (NWU) has recently emerged as an attractive quantitative imaging biomarker ${ }^{11-13}$. Not only is it easily and quickly assessed, but it is based on pathophysiological processes leading to increased vasogenic edema and, as a result, is specific for irreversible tissue injury. When taken together, NWU and CTP-based core estimation parameters could provide a more accurate picture of both lesion size and stage of infarction at the admission timepoint. We hypothesize that low levels of NWU are predictive of overestimation within the CTP-defined core lesion.

\section{Results}

\section{Patient Characteristics}

284 patients fulfilled the inclusion criteria. The median age was 76.5 years (IQR: $65-82$ ) and $48 \%$ (135) were female. The median pCore was $11.9 \mathrm{~mL}$ (IQR: 3.6 - 31.1), measured after a median time from onset to imaging of 3.2 hours (IQR: $1.3-4.7$ ). A median NWU of $7.2 \%$ (IQR: $2.6-12.8$ ) was observed and the cohort had a median lesion growth of $18.4 \mathrm{ml}$ (IQR: -1.8 - 118.8). 49.3\% (138/280) underwent successful reperfusion, defined as an expanded Thrombolysis in Cerebral Infarction (eTICI) score of 2c/3. Median FIV was $32.8 \mathrm{~mL}$ (IQR: 5.7 - 146.2). Good outcome was observed in 29.3\%. Patients with pCore overestimation had significantly higher ASPECTS (8 versus 7), lower NWU (5.6\% versus $7.6 \%$ ), larger pCores (34.2 mL versus $8.7 \mathrm{~mL}$ ), and lower rates of successful reperfusion (39.5\% versus $51 \%$ ) (Table 1 ). For baseline characteristics of the entire cohort and stratified according to pCore volume, see

\section{Supplementary Tables S1 and S2.}


Table 1

Baseline, imaging, and procedural characteristics of the study sample $(n=284)$, stratified by core overestimation.

\begin{tabular}{|c|c|c|}
\hline Variable* & $\begin{array}{l}\text { Core Overestimation - Yes } \\
(n=45)\end{array}$ & $\begin{array}{l}\text { Core Overestimation - No ( } \mathrm{n} \\
=229 \text { ) }\end{array}$ \\
\hline Age (years) - median (IQR) & $78(67-82)$ & $76(64-82)$ \\
\hline $\begin{array}{l}\text { mRS before admission - median } \\
\text { (IQR) }\end{array}$ & $0(0-2), n=39$ & $0(0-1), n=189$ \\
\hline NIHSS - median (IQR) & $16(15-29)$ & $15(11-19), n=220$ \\
\hline $\begin{array}{l}\text { Systolic blood pressure }(\mathrm{mmHg}) \text { - } \\
\text { mean (SD) }\end{array}$ & $158(35)$ & $161(28), n=219$ \\
\hline $\begin{array}{l}\text { Diastolic blood pressure }(\mathrm{mmHg}) \text { - } \\
\text { mean (SD) }\end{array}$ & $86(18)$ & $86(15), n=217$ \\
\hline 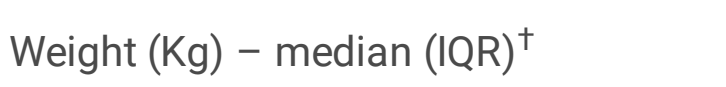 & $70(62-80), n=38$ & $80(70-89), n=164$ \\
\hline ASPECTS - median (IQR) ${ }^{\dagger}$ & $8(7-9)$ & $7(6-9), n=228$ \\
\hline NWU $(\%)-$ median $(\mathrm{IQR})^{+}$ & $5.6(1.3-7.8)$ & $7.6(2.8-13.7)$ \\
\hline pCore $(\mathrm{mL})-\operatorname{median}(\mathrm{IQR})^{\dagger}$ & $34.2(25.0-67.2)$ & $8.7(2.3-25.6)$ \\
\hline Penumbra (mL) - median (IQR) & $83.9(49.1-141.1)$ & $70.6(43.6-105.9)$ \\
\hline $\begin{array}{l}\text { Successful reperfusion }(\mathrm{eTICl} 2 \mathrm{c} / 3)- \\
\mathrm{n}(\%)^{\dagger}\end{array}$ & $17(39.5 \%)$ & $121(51 \%)$ \\
\hline mRS90 0-2 - n (\%) & $16(42.1 \%), n=38$ & $47(27.0 \%), n=174$ \\
\hline \multicolumn{3}{|c|}{ * $\mathrm{n}$ is provided only in case of missing values } \\
\hline \multicolumn{3}{|l|}{${ }^{\dagger}$ Wilcoxon rank sum test $p<0.05$} \\
\hline $\begin{array}{l}\text { IQR: interquartile range; mRS: modified } \\
\text { ASPECTS: Alberta Stroke Program Early } \\
\text { volume, eTICl: expanded Thrombolysis }\end{array}$ & $\begin{array}{l}\text { ankin scale; NIHSS: National } \\
\text { CT Score; NWU: net water upt } \\
\text { n Cerebral Infarction scale }\end{array}$ & $\begin{array}{l}\text { titutes of Health Stroke Scale; } \\
\text { e; pCore: CTP-defined core }\end{array}$ \\
\hline
\end{tabular}

\section{Association of variables with pCore overestimation}

In univariable logistic regression, ASPECTS (RR 1.21, 95\%Cl: 1.05 - 1.39), pCore (RR 1.01, 95\%Cl: 1.011.02), NWU (RR 0.93, 95\%Cl: $0.90-0.97)$, onset to imaging (RR 1.00, 95\% Cl: $0.99-1.00)$, and onset to recanalization (RR $0.99,95 \% \mathrm{Cl}: 0.99-1.00$ ) were significantly associated with core overestimation (Figure 1, Table 2). These associations remained significant in multivariable regression modelling (Table 2). 
Table 2

Univariable and multivariable binary logistic regression analysis of the effect of baseline, imaging, and procedural variables on pCore overestimation and good functional outcome (mRS 0-2).

\begin{tabular}{|c|c|c|c|c|}
\hline \multirow[b]{2}{*}{ Predictor } & \multicolumn{2}{|c|}{ Core overestimation } & \multicolumn{2}{|c|}{ Good outcome (mRS 0-2) at 90 days } \\
\hline & $\begin{array}{l}\text { Unadjusted } \\
\text { RR }\end{array}$ & $\begin{array}{l}\text { Adjusted } \\
\mathrm{RR}^{\star}\end{array}$ & $\begin{array}{l}\text { Unadjusted RR } \\
(95 \% \mathrm{Cl})\end{array}$ & $\begin{array}{l}\text { Adjusted RR* } \\
(95 \% \mathrm{Cl})\end{array}$ \\
\hline & $(95 \% \mathrm{Cl})$ & $(95 \% \mathrm{Cl})$ & & \\
\hline Age (years) & $\begin{array}{l}1.02(0.99- \\
1.04)\end{array}$ & - & $0.97(0.96-0.98)$ & $0.97(0.96-0.98)$ \\
\hline Sex & $\begin{array}{l}0.79(0.46- \\
1.35)\end{array}$ & - & $1.27(0.83-1.94)$ & - \\
\hline NIHSS & $\begin{array}{l}1.02(0.99- \\
1.05)\end{array}$ & - & $0.93(0.90-0.96)$ & $0.95(0.92-0.99)$ \\
\hline ASPECTS & $\begin{array}{l}1.21(1.05- \\
1.39)\end{array}$ & $\begin{array}{l}1.28(1.09- \\
1.51)\end{array}$ & $1.2(1.11-1.37)$ & $1.18(1.06-1.31)$ \\
\hline pCore & $\begin{array}{l}1.01(1.01- \\
1.02)\end{array}$ & $\begin{array}{l}1.02(1.01- \\
1.02)\end{array}$ & $0.99(0.98-1.00)$ & - \\
\hline NWU (\%) & $\begin{array}{l}0.93(0.90- \\
0.97)\end{array}$ & $\begin{array}{l}0.94(0.89- \\
0.98)\end{array}$ & $0.98(0.95-1.01)$ & - \\
\hline Intravenous alteplase & $\begin{array}{l}1.23(0.76- \\
1.96)\end{array}$ & - & $1.55(0.98-2.45)$ & - \\
\hline Onset to imaging (min) & $\begin{array}{l}1.00(0.99- \\
1.00)^{\S}\end{array}$ & $\begin{array}{l}1.00(1.00- \\
1.00)^{\S}\end{array}$ & $1.00(1.00-1.00)$ & - \\
\hline $\begin{array}{l}\text { Onset to recanalization } \\
\text { (min) }\end{array}$ & $\begin{array}{l}1.00(0.99- \\
1.00)^{\S}\end{array}$ & $\begin{array}{l}1.00(0.99- \\
1.00)^{\S}\end{array}$ & $1.00(1.00-1.00)$ & - \\
\hline $\begin{array}{l}\text { Successful reperfusion } \\
(\mathrm{eTICl} 2 \mathrm{c} / 3 \text { ) }\end{array}$ & $\begin{array}{l}1.18(0.69- \\
2.01)\end{array}$ & - & $1.61(1.05-2.46)$ & $1.55(1.06-2.28)$ \\
\hline \multicolumn{5}{|c|}{ *Significant predictors in univariable regression were used to build the multivariable model } \\
\hline \multicolumn{5}{|c|}{$\S$ Values are rounded to the nearest decimal } \\
\hline \multicolumn{5}{|c|}{ Significant associations are shown in bold } \\
\hline $\begin{array}{l}\text { mRS: modified Rankin } \\
\text { ASPECTS: Alberta Strok } \\
\text { Thrombolysis in Cerebra }\end{array}$ & $\begin{array}{l}\text { RR: relative ris } \\
\text { ram Early CT } \\
\text { rction }\end{array}$ & $\begin{array}{l}\text { NIHSS: Natiol } \\
\text { ore; NWU: ne }\end{array}$ & $\begin{array}{l}\text { Institutes of Healt } \\
\text { ater uptake; eTICl: }\end{array}$ & $\begin{array}{l}\text { roke Scale; } \\
\text { anded }\end{array}$ \\
\hline
\end{tabular}

Following stratification, an association between age, ASPECTS, eTICl grade, pCore, and NWU and core overestimation was observed in the subgroup of patients with a pCore $\geq 50 \mathrm{~mL}$, while NIHSS, pCore, and NWU were the only significant predictors of core overestimation in the small core (pCore $<50 \mathrm{~mL}$ ) subgroup (Figures 2 and 3, Supplementary Table S3). 


\section{Association of variables with favorable outcome, mRS 0-2 at 90 days}

Univariable logistic regression revealed age (RR 0.97, 95\%Cl: 0.96-0.98), NIHSS (RR 0.93, 95\%Cl: 0.900.96), ASPECTS (RR 1.2, 95\%Cl: 1.11-1.37), and successful reperfusion (RR 1.61,95\%Cl: 1.05-2.46) to be significantly associated with favorable outcome at 90 days; these associations remained stable in a multivariable logistic regression model including all the above parameters and following adjustment for sex (Table 2).

In the small core subgroup (pCore $<50 \mathrm{~mL}$ ), there were no differences in the variables that were observed to be associated with an mRS90 of 0-2 for the overall patient sample. For those with a pCore $\geq 50 \mathrm{~mL}$, however, only age was a significant predictor of favorable outcome (Supplementary Table S4).

\section{Association of variables with lesion growth}

Significant associations were observed for ASPECTS, successful recanalization, NWU, pCore, and the cubic root of lesion growth (Table 3). Linear regression analysis showed that increasing NWU led to an increase in the cubic root of lesion growth ( $\mathrm{B} 0.06,95 \% \mathrm{Cl}: 0.02-0.09, \mathrm{p}=0.001$, Figure 4, Table 3 ). The sensitivity analysis showed that ASPECTS, NWU, and successful reperfusion were significantly associated with lesion growth in both the large and small core subgroups (Supplementary Table S5). 
Table 3

Univariable and multivariable linear regression analysis of baseline, imaging, and procedural variables with the cubic root of lesion growth.

\begin{tabular}{|lll|}
\hline Variable & B-Coefficient (95\% Cl) & Adjusted B-coefficient* (95\% Cl) \\
\hline Age (years) & $-0.01(-0.04-0.01)$ & - \\
\hline Sex & $0.39(-0.15-0.92)$ & - \\
\hline NIHSS & $-0.001(-0.04-0.04)$ & - \\
\hline ASPECTS & $-0.33(-0.46-[-0.20])$ & $-0.43(-0.57-[-0.30])$ \\
\hline NWU (\%) & $0.06(0.02-0.09)$ & $0.04(0.01-0.07)$ \\
\hline pCore & $-0.01(-0.02-[-0.002])$ & $-0.02(-0.03-[-0.01])$ \\
\hline Time onset to imaging (min) & $0.001(-0.0003-0.002)$ & - \\
\hline Time onset to recanalization (mins) & $0.001(-0.001-0.002)$ & - \\
\hline Successful reperfusion (eTICI 2c/3) & $-0.87(-1.39-[-0.34])$ & $-0.86(-1.33-[-0.38])$ \\
\hline *Significant predictors in univariable regression were used to build the multivariable model \\
\hline Significant associations are shown in bold
\end{tabular}

\section{Discussion}

The purpose of this study was to investigate the relationship between NWU on admission imaging and the probability of CTP-based ischemic core overestimation. We hypothesized that patients with large cores, yet low NWU were the most likely to show core overestimation. The main findings of this study are shown Figure 2; in this patient cohort, low NWU was indeed significantly associated with core overestimation, thus supporting our hypothesis. Interestingly, this relationship appeared to be particularly robust for the subgroup of patients with large baseline ischemic cores. We also observed that patients with higher levels of NWU on admission imaging had increased lesion growth, although this relationship was no longer significant following adjustment for patient baseline characteristics and reperfusion status. Despite this, these data support a more granular depiction of lesion growth dynamics and propose a multi-stage chronological approach to ischemic core lesion imaging, based on known metabolic processes. Initially, the cells distal to the arterial occlusion undergo oxygen deprivation, resulting in a compensatory increase in CBF. Over time, however, these compensatory mechanisms fail, leading to a progressive decline in $\mathrm{CBF}$ and $\mathrm{CBV}$, with transformation of the penumbra into irreversibly damaged tissue. Due to continued cellular oxygen deprivation, disruption of the blood brain barrier ensues, leading to the influx of ions and subsequently tissue water uptake. The combined assessment of CTP parameters and NWU could reflect these stages; in patients with large CBV deficits, but low NWU, the compensatory 
mechanisms are largely maintained - the core lesion is "reversible". On the other hand, those with large CBV lesions and high NWU have more likely crossed the threshold into irreversible tissue damage. It is possible that these patients also experience more growth due to a developing increase of NWU within "borderline" penumbral tissue, signaling initial breakdown of the blood-brain-barrier, which was not assessed in this study. Furthermore, the current definition of core volume is based on binary thresholding, thereby likely oversimplifying complex pathophysiological processes ${ }^{14}$. It would be interesting, therefore, to examine whether there is a "gradient" of NWU around the CBV-derived core lesion.

The associations of age, NIHSS, ASPECTS, and successful reperfusion with favorable outcome confirm that which has been extensively shown in the literature ${ }^{15-17}$. While NWU was not a significant predictor of mRS90 0-2 in this patient cohort, its potential role in the improved selection of patients most likely to benefit from EVT would be of substantial value, particularly for those who would have otherwise been excluded due to the results of advanced imaging. Indeed, ASPECTS represents a binary measure of early ischemic changes, not taking the degree of hypodensity into account. Performing concomitant NWU analyses could provide a more detailed overview of the extent of tissue damage at the time of baseline imaging.

Patients with large areas of severe hypoattenuation (i.e., high water uptake) are frequently excluded from EVT, and thus underrepresented in this study. Nevertheless, in a sensitivity analysis restricted to the patient subgroup with a pCore $\geq 50 \mathrm{~mL}$, successful reperfusion was found to be negatively associated with lesion growth, with younger patients more likely to achieve a good outcome. This points toward a potential benefit of EVT, even for those with large baseline infarctions. The currently running randomized trials, including TESLA (ClinicalTrials.gov Identifier: NCT03805308), TENSION (ClinicalTrials.gov Identifier: NCT03094715), IN-EXTREMIS-LASTE (ClinicalTrials.gov Identifier: NCT03811769), and SELECT2 (ClinicalTrials.gov Identifier: NCT03876457) will hopefully soon provide evidence for such cases.

This study has limitations. First, despite the long recruitment period, relatively few patients were included. This was particularly true for the large core subgroup, as such patients rarely undergo EVT. This likely resulted in the sensitivity analysis being underpowered to detect potential predictors of outcome and lesion growth. Nevertheless, we believe the significant association between NWU and core overestimation to be of particular interest for this patient population. Second, our standard acute stroke protocol consists of NCCT, CTA, and CTP independent of time window (i.e., is also done for patients presenting within 6 hours). This should lend caution to stroke physicians in their decision-making processes, as the use of advanced imaging in the early time window could result in false exclusion of patients. Third, the association between patient clinical, imaging, and procedural characteristics with lesion growth may have been confounded by the potentially inaccurate measurements of pCore. To this end, we adjusted the analyses for the initial core measurements. Fourth, we included patients with both successful (eTICl $2 c / 3$ ) and unsuccessful reperfusion in our cohort, which likely impacted infarct growth dynamics.

To our knowledge, this is the first study that examines the modifying effect of NWU on CTP-derived core overestimation in patients with AIS who underwent EVT. This relationship of NWU on infarct core lesion 
size and growth is particularly relevant for those subsets of patients that have been, if inadvertently, excluded from the recent large clinical trials. If CTP-based parameters are to remain a major part of the decision-making workflow in AIS, a certain level of accuracy must be achieved. The relatively easy adjunct calculation of NWU could help clinicians decide which patients would most likely benefit from EVT thereby further contributing to the personalization of stroke medicine.

\section{Conclusion}

NWU can be used as a supplemental tool to CTP during admission imaging to more accurately assess the extent of irreversible ischemia, particularly relevant for patients with large CTP-defined cores who would otherwise be excluded from treatment.

\section{Methods}

\section{Study Population}

This represents a single-center retrospective observational cohort study of patients suffering from AIS due to LVO, consecutively treated at the University Medical Center Hamburg-Eppendorf between June 2015 and July 2020. Patient selection was done based on the following a priori defined inclusion criteria: (1) age > 18 years, (2) multimodal CT (non-contrast CT (NCCT), CT angiography (CTA), and CTP) performed at admission; (3) absence of intracranial hemorrhage (4) endovascular treatment with or without prior intravenous (i.v.) recombinant tissue plasminogen activator (rtPA); and (5) follow-up imaging (NCCT) performed within 24 hours of admission. Patient baseline characteristics were extracted from the medical records and clinical outcome parameters (modified Rankin scale (mRS) at discharge and 90 days, mRS90) were documented, if available. The local ethics review board of the University Medical Center Hamburg-Eppendorf approved the use of anonymized patient data for this retrospective analysis and waived the requirement for informed consent. All study protocols and procedures were conducted in accordance with ethical guidelines of the University Medical Center Hamburg-Eppendorf and in compliance with the Declaration of Helsinki.

\section{Image Acquisition}

All patients received multimodal stroke imaging at admission with NCCT, CTA, and CTP

performed in equal order on 256 or 384 dual slice scanners (Philips iCT 256, Siemens Somatom Force). NCCT: $120 \mathrm{kV}, 280$ to $340 \mathrm{~mA}, 5.0 \mathrm{~mm}$ slice reconstruction, 1-mm increment; CTA: 100kV, 260 to $300 \mathrm{~mA}$, $5.0 \mathrm{~mm}$ slice reconstruction, $1-\mathrm{mm}$ increment, $80 \mathrm{~mL}$ highly iodinated contrast medium and $50 \mathrm{~mL} \mathrm{NaCl}$ flush at $4 \mathrm{~mL} /$ second; CTP: $80 \mathrm{kV}, 200$ to $250 \mathrm{~mA}, 5 \mathrm{~mm}$ slice reconstruction (maximum $10 \mathrm{~mm}$ ), slice sampling rate $1.50 \mathrm{~s}$ (minimum $1.33 \mathrm{~s}$ ), scan time $45 \mathrm{~s}$ (maximum 60s), biphasic injection with $30 \mathrm{~mL}$ (maximum $40 \mathrm{~mL}$ ) of highly iodinated contrast medium with $350 \mathrm{mg}$ iodine $/ \mathrm{mL}$ (maximum $400 \mathrm{mg} / \mathrm{mL}$ ) injected with at least $4 \mathrm{~mL} / \mathrm{s}$ (maximum $6 \mathrm{~mL} / \mathrm{s}$ ) followed by $30 \mathrm{~mL}$ sodium chloride chaser bolus, whole- 
brain coverage of $12 \mathrm{~cm}$. All perfusion datasets underwent quality control and were excluded in case of severe motion artifacts.

\section{Image Analysis}

Anonymized CT imaging data was segmented manually using semiautomatic commercially available software (Analyze 11.0, Biomedical Imaging Resource, Mayo Clinic, Rochester, MN). The raters (RM, SE) were blinded for all other imaging data and patient information. CTP-guided delineation of ischemic lesion NWU on admission imaging was determined according to recently published methods ${ }^{11,18}$. In brief, the early hypoattenuated ischemic core lesion in NCCT was assessed by densitometric measurements $\left(D_{\text {ischemic }}\right)$. A similarly drawn region of interest was placed on the contralateral, non-affected hemisphere $\left(D_{\text {normal }}\right)$. Region of interest (ROI) histograms were sampled between 20 and 80 Hounsfield units $(H U)$ to exclude voxels that correspond to cerebrospinal fluid or calcifications. The density measurements, $D_{\text {infarct }}$ and $D_{\text {normal }}$, were then used to calculate percent NWU (\%NWU, Equation 1), i.e., the proportion of water uptake within the infarct lesion compared to the contralateral side.

$\%$ NWU $=\left(1-D_{\text {ischemic }} / D_{\text {norma }}\right) \times 100 \%$

Raw perfusion data were analyzed on a Siemens ${ }^{\grave{o}}$ workstation using Syngo ${ }^{\circ}$ VPCT Neuro software (Siemens Healthcare, Erlangen, Germany). Quantitative maps of relative CBF (rCBF), CBV, MTT, and Tmax were generated using a delay-insensitive algorithm. For comparability, we selected a predicted core (pCore) threshold of $\mathrm{rCBF} \leq 20 \%$, as this has been described to have the highest level of agreement with RAPID-generated rCBF-based volumes ${ }^{19}$. Hypoperfusion volume was determined to be the volume of tissue with a prolonged Tmax of at least 6 seconds (Tmax $>6 \mathrm{~s}$ ). Mismatch (penumbral) volume was defined as the difference between this volume and pCore.

The final infarct lesion volume (FIV) on follow-up imaging (NCCT) was determined by manual segmentation using semiautomatic commercially available software (Analyze 11.0, Biomedical Imaging Resource, Mayo Clinic, Rochester, MN). Finally, lesion growth between admission and follow-up imaging was determined by calculating the difference between the FIV and pCore (Equation 2).

Lesion growth $=$ FIV - pCore $(2)$

\section{Statistical Analysis and Outcome Measures}

Descriptive analyses were used to define population baseline, imaging, and procedural characteristics. Shapiro-Wilk and histograms tested for the normality of distributions. The primary outcome measure was the binarized core overestimation variable; if lesion growth was negative by more than $10 \mathrm{ml}$ (chosen in accordance with a previous study ${ }^{9}$ and to account for segmentation error), the pCore was scored as being overestimated. The secondary outcomes were good functional outcome at 90 days, mRS90 0-2, and lesion growth. Because the volumetric measurements of lesion growth were heavily skewed, the cubic root was taken for the analysis. Linear (cubic root of lesion growth) and binary logistic regression models 
(core overestimation and favorable outcome, mRS90 0-2) were used to test the interactions. Significant predictors of outcome variables in univariable regression were used to construct the multivariable models. A sensitivity analysis was performed following stratification of the cohort according to pCore volume $\geq 50 \mathrm{~mL}$ vs. $<50 \mathrm{~mL}$. This cut off was chosen to be in line with the inclusion criteria of multiple prospective clinical trials, as well as observations from the HERMES metaanalysis $2,20,21$.

All analyses were performed using Stata 15.1 (StataCorp LLC). Normally distributed variables are displayed as mean and standard deviation (SD). Non-normally distributed data are displayed as median and interquartile range (IQR). Categorical variables are reported as proportions. Binary logistic regression results are presented as relative risks (RR) with $95 \%$ confidence intervals $(95 \% \mathrm{Cl})$. P-values $<0.05$ were considered significant.

\section{Declarations}

\section{DATA AVAILABILITY}

Data are available from the corresponding author upon reasonable request.

\section{AUTHOR CONTRIBUTIONS}

RM: Study design. Acquisition of data. Image processing. Image analysis. Data analysis. Statistical analysis. Drafting the manuscript and revising it critically.

SE: Study design. Acquisition of data. Image processing. Image analysis. Data analysis. Drafting the manuscript and revising it critically.

LM: Data analysis. Drafting the manuscript and revising it critically.

TE: Data analysis. Drafting the manuscript and revising it critically.

MB: Data analysis. Drafting the manuscript and revising it critically.

HK: Data analysis. Drafting the manuscript and revising it critically.

MTN: Data analysis. Drafting the manuscript and revising it critically.

TDF: Data analysis. Drafting the manuscript and revising it critically.

GS: Data analysis. Statistical analysis. Drafting the manuscript and revising it critically.

GT: Data analysis. Drafting the manuscript and revising it critically.

JF: Study design. Data analysis. Drafting the manuscript and revising it critically.

UH: Study design. Data analysis. Drafting the manuscript and revising it critically. 
AK: Study design. Data analysis. Drafting the manuscript and revising it critically.

GB: Study design. Acquisition of data. Image processing. Image analysis. Data analysis. Statistical analysis. Drafting the manuscript and revising it critically.

\section{COMPETING INTERESTS}

Jens Fiehler: Consultant for Acandis, Boehringer Ingelheim, Codman, Microvention, Sequent, Stryker. Speaker for Bayer Healthcare, Bracco, Covidien/ev3, Penumbra, Philips, Siemens. Grants from Bundesministerium für Wirtschaft und Energie (BMWi), Bundesministerium für Bildung und Forschung (BMBF), Deutsche Forschungsgemeinschaft (DFG), European Union (EU), Covidien, Stryker (THRILL study), Microvention (ERASER study), Philips.

Götz Thomalla: Has received grant support and lecture fees from Bayer, personal fees from Acandis, Boehringer Ingelheim, Bristol-Myers Squibb/Pfizer, and Daiichi Sankyo, Portola, and Stryker.

Andre Kemmling: Research collaboration agreement: Siemens Healthcare.

All other authors declare that they have no conflicts of interest.

\section{References}

1. Goyal, M., Menon, B. K., Almekhlafi, M. A., Demchuk, A. \& Hill, M. D. The Need for Better Data on Patients with Acute Stroke Who Are Not Treated Because of Unfavorable Imaging. American Journal of Neuroradiology 38, 424-425, doi:10.3174/ajnr.A5094 (2017).

2. Nogueira, R. G. et al. Thrombectomy 6 to 24 Hours after Stroke with a Mismatch between Deficit and Infarct. N Engl J Med 378, 11-21, doi:10.1056/NEJMoa1706442 (2018).

3. Albers, G. W. et al. Thrombectomy for Stroke at 6 to 16 Hours with Selection by Perfusion Imaging. $N$ Engl J Med 378, 708-718, doi:10.1056/NEJMoa1713973 (2018).

4. Rocha, M. \& Jovin, T. G. Fast Versus Slow Progressors of Infarct Growth in Large Vessel Occlusion Stroke: Clinical and Research Implications. Stroke 48, 2621-2627, doi:10.1161/STROKEAHA.117.017673 (2017).

5. González, R. G. et al. Identifying Severe Stroke Patients Likely to Benefit From Thrombectomy Despite Delays of up to a Day. Scientific Reports 10, 4008, doi:10.1038/s41598-020-60933-3 (2020).

6. Powers William, J. et al. Guidelines for the Early Management of Patients With Acute Ischemic Stroke: 2019 Update to the 2018 Guidelines for the Early Management of Acute Ischemic Stroke: A Guideline for Healthcare Professionals From the American Heart Association/American Stroke Association. Stroke 50, e344-e418, doi:10.1161/STR.0000000000000211 (2019). 
7. Albers, G. W. Use of Imaging to Select Patients for Late Window Endovascular Therapy. Stroke 49, 2256-2260, doi:doi:10.1161/STROKEAHA.118.021011 (2018).

8. Boned, S. et al. Admission CT perfusion may overestimate initial infarct core: the ghost infarct core concept. Journal of Neurolnterventional Surgery 9, 66-69, doi:10.1136/neurintsurg-2016-012494 (2017).

9. Martins, N. et al. Ghost Infarct Core and Admission Computed Tomography Perfusion: Redefining the Role of Neuroimaging in Acute Ischemic Stroke. Intervent Neurol 7, 513-521, doi:10.1159/000490117 (2018).

10. Rotem, S. H. et al. Infarct Core Reliability by CT Perfusion is a Time-Dependent Phenomenon. J Neuroimaging 30, 240-245, doi:10.1111/jon.12692 (2020).

11. Broocks, G. et al. Computed Tomography-Based Imaging of Voxel-Wise Lesion Water Uptake in Ischemic Brain: Relationship Between Density and Direct Volumetry. Investigative Radiology 53, 207213, doi:10.1097/RLI.0000000000000430 (2018).

12. Broocks, G. et al. Ischemic Lesion Growth in Acute Stroke: Water Uptake Quantification Distinguishes Between Edema and Tissue Infarct. J Cereb Blood Flow Metab (2019).

13. Broocks, G. et al. Ischemic lesion growth in acute stroke: Water uptake quantification distinguishes between edema and tissue infarct. J Cereb Blood Flow Metab, 271678X19848505, doi:10.1177/0271678X19848505 (2019).

14. Flottmann, F. et al. CT-perfusion stroke imaging: a threshold free probabilistic approach to predict infarct volume compared to traditional ischemic thresholds. Sci Rep 7, 6679, doi:10.1038/s41598017-06882-w (2017).

15. Baird, A. E., Warach, S. \& Dambrosia, J. Prediction of outcome after stroke. The Lancet 358, 15531554 (2001).

16. Hand, P., Wardlaw, J., Lindley, R. \& Keir, S. Prediction of outcome after stroke. The Lancet 358, 15521553 (2001).

17. König, I. R. et al. Predicting Long-Term Outcome After Acute Ischemic Stroke. Stroke 39, 1821-1826, doi:doi:10.1161/STROKEAHA.107.505867 (2008).

18. Broocks, G. et al. Quantitative Lesion Water Uptake in Acute Stroke Computed Tomography is a Predictor of Malignant Infarction. Stroke 49, 1906-1912, doi:10.1161/STROKEAHA.118.020507 (2018).

19. Bathla, G. et al. Achieving comparable perfusion results across vendors. The next step in standardizing stroke care: a technical report. Journal of Neurointerventional Surgery 11, 1257-1260, doi:10.1136/neurintsurg-2019-014810 (2019).

20. Saver, J. L. et al. Stent-retriever thrombectomy after intravenous t-PA vs. t-PA alone in stroke. N Engl J Med 372, 2285-2295, doi:10.1056/NEJMoa1415061 (2015).

21. Boers, A. M. M. et al. Association of follow-up infarct volume with functional outcome in acute ischemic stroke: a pooled analysis of seven randomized trials. J Neurointerv Surg 10, 1137-1142, doi:10.1136/neurintsurg-2017-013724 (2018). 
Figures

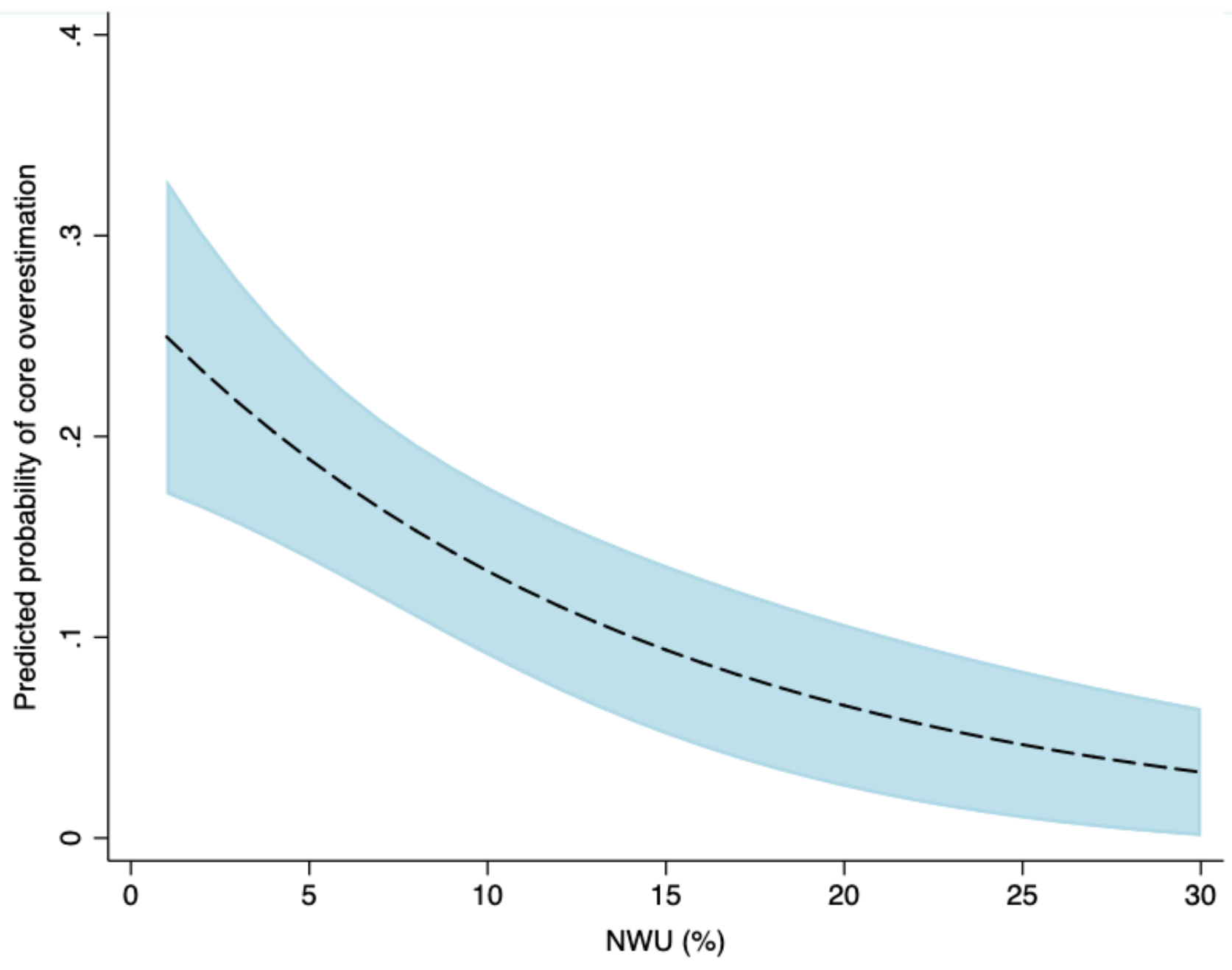

Figure 1

Predicted probability of core overestimation by NWU at admission. Lower levels of NWU were significantly and positively associated with core overestimation. NWU: net water uptake. 


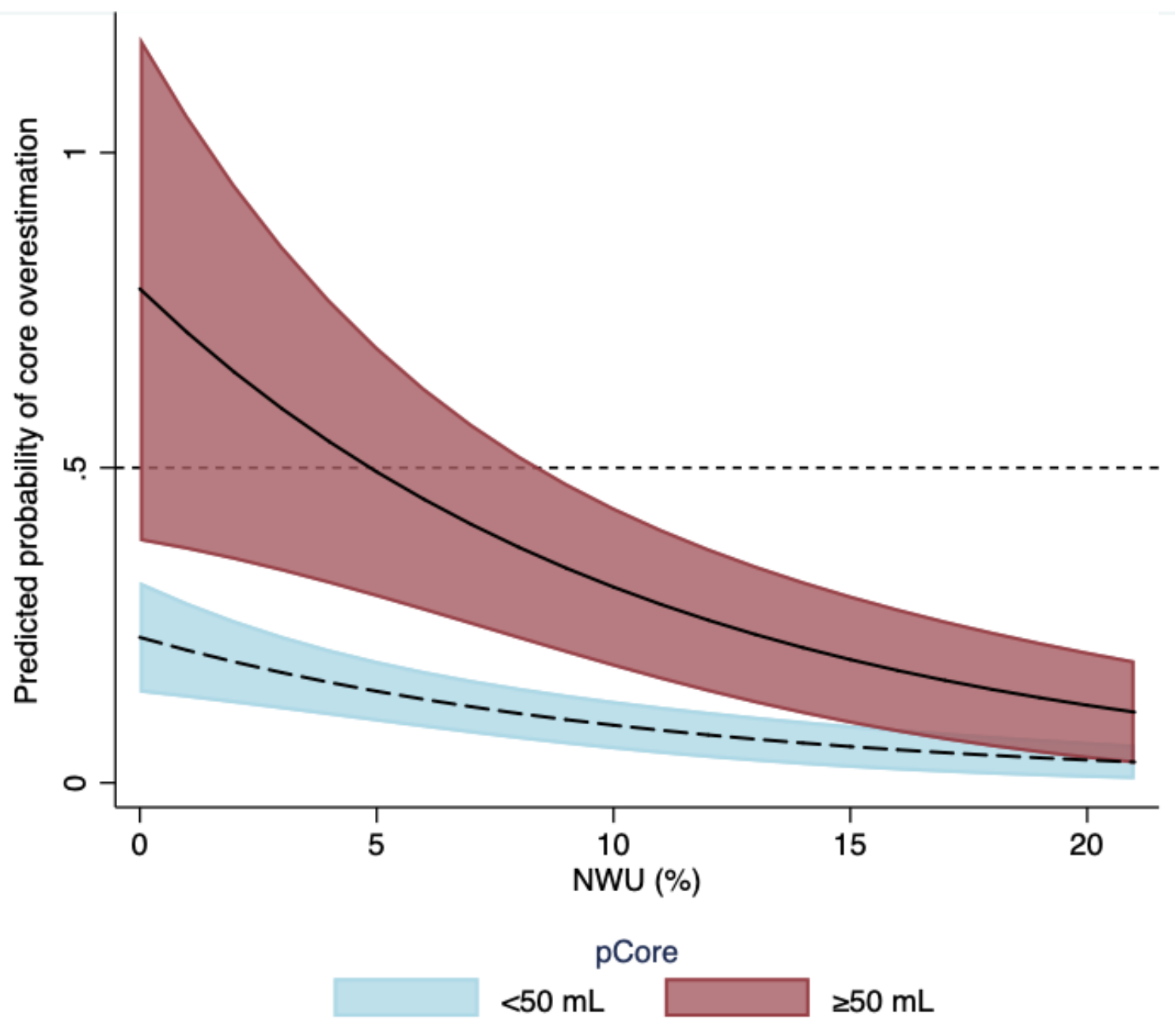

Figure 2

Predicted probability of core overestimation by NWU at admission, stratified by pCore volume. Lower levels of NWU were significantly and positively associated with core overestimation, particularly for those patients with a large pCore $\geq 50 \mathrm{~mL}$. NWU: net water uptake. 

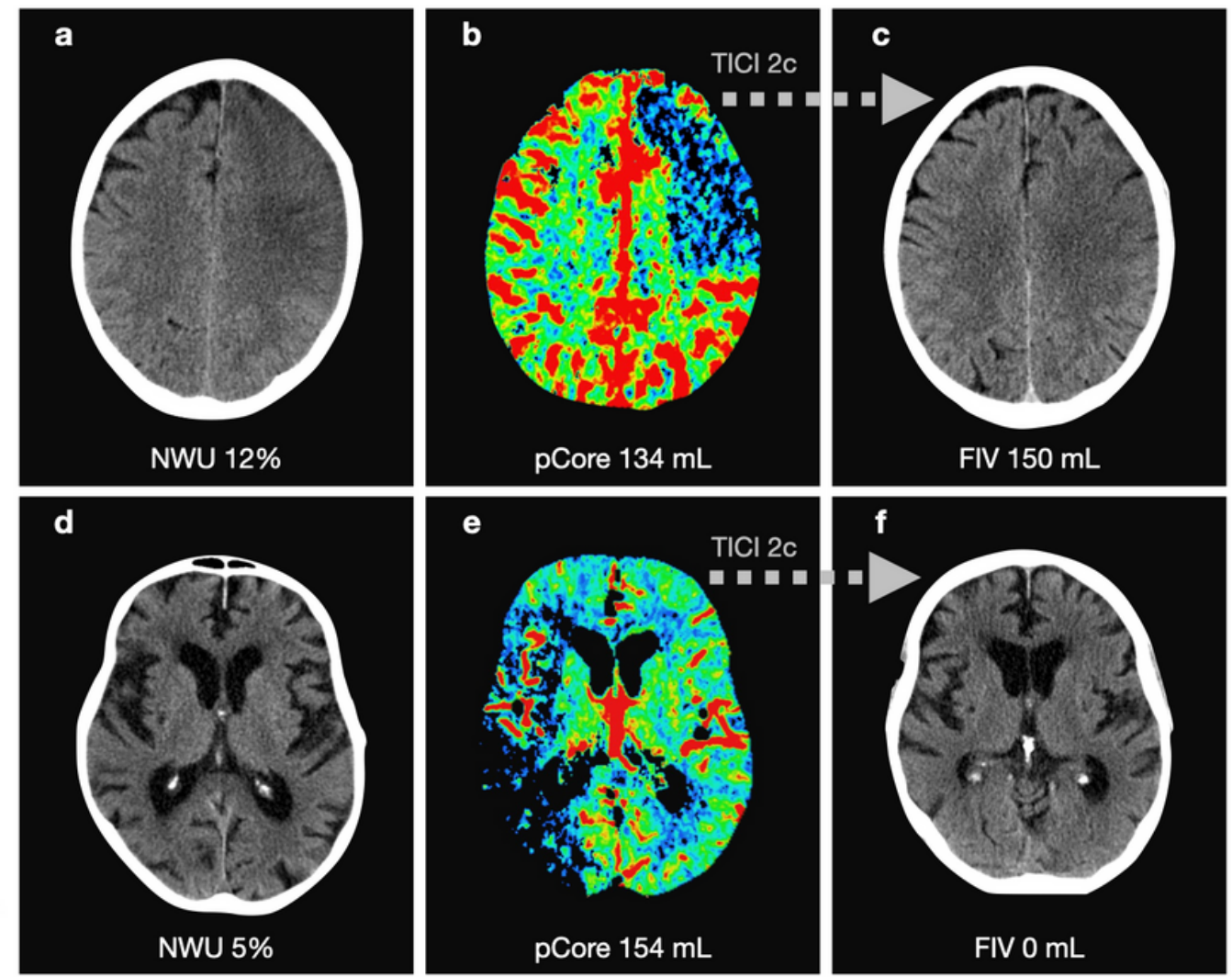

Figure 3

Examples of patients with high (A-C) and low (D-F) NWU and their association with pCore overestimation. Both patients presented within a similar time frame and achieved near-completed reperfusion. (A, D: admission imaging; $\mathrm{B}, \mathrm{E}$ : $\mathrm{rCBF}<20 \%$ CTP maps; C, F: follow-up imaging). NWU: net water uptake; pCore: rCBF20\%-defined core at admission; CTP: CT perfusion. 


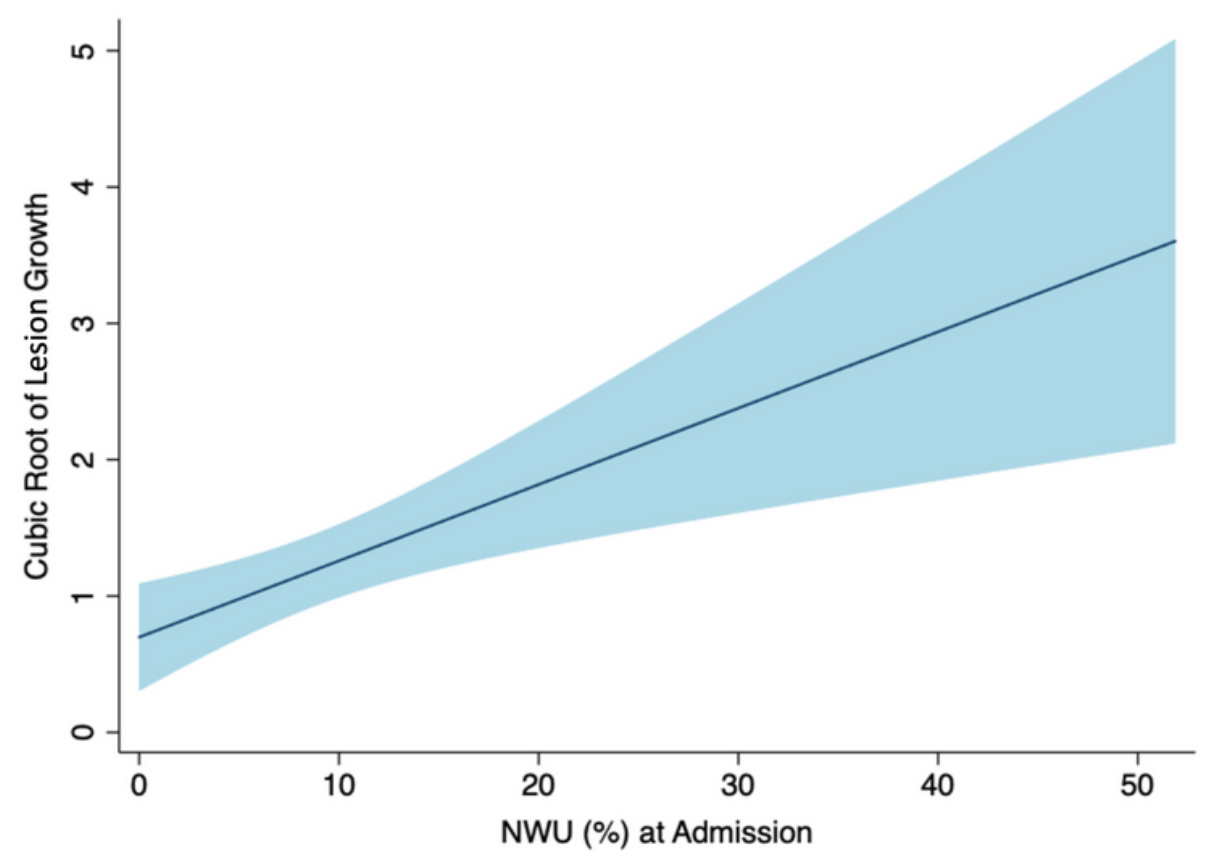

Figure 4

Relationship between NWU and lesion growth. Lower NWU was significantly associated with less lesion growth. NWU: net water uptake.

\section{Supplementary Files}

This is a list of supplementary files associated with this preprint. Click to download.

- SupplementaryMaterial.pdf 